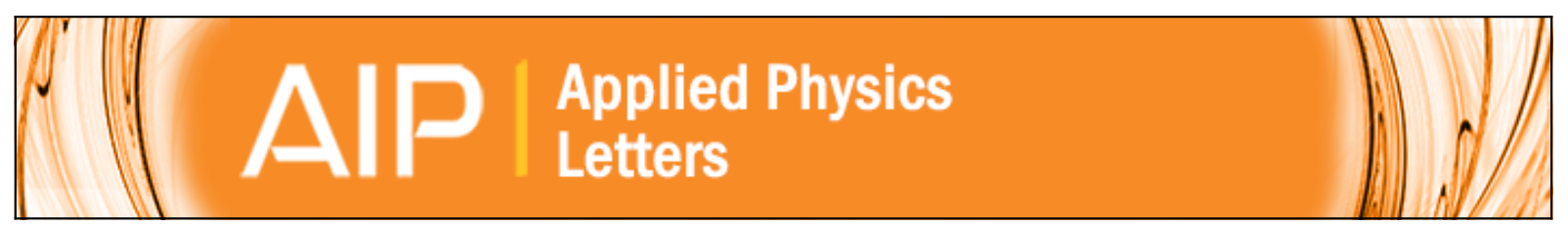

\title{
Laser material processing with tightly focused cylindrical vector beams
}

Rokas Drevinskas, Jingyu Zhang, Martynas Beresna, Mindaugas Gecevičius, Andrey G. Kazanskii, Yuri P.

Svirko, and Peter G. Kazansky

Citation: Applied Physics Letters 108, 221107 (2016); doi: 10.1063/1.4953455

View online: http://dx.doi.org/10.1063/1.4953455

View Table of Contents: http://scitation.aip.org/content/aip/journal/apl/108/22?ver=pdfcov

Published by the AIP Publishing

\section{Articles you may be interested in}

Influence of intermediate layers on the surface condition of laser crystallized silicon thin films and solar cell performance

J. Appl. Phys. 119, 045306 (2016); 10.1063/1.4940953

Dynamical process of $\mathrm{KrF}$ pulsed excimer laser crystallization of ultrathin amorphous silicon films to form $\mathrm{Si}$ nano-dots

J. Appl. Phys. 111, 094320 (2012); 10.1063/1.4716467

Crystallization and surface texturing of amorphous-Si induced by UV laser for photovoltaic application

J. Appl. Phys. 111, 043106 (2012); 10.1063/1.3686612

Altering the nucleation of thermally annealed hydrogenated amorphous silicon with laser processing Appl. Phys. Lett. 95, 251902 (2009); 10.1063/1.3259654

Thickness dependence of properties of excimer laser crystallized nano-polycrystalline silicon

J. Appl. Phys. 97, 114305 (2005); 10.1063/1.1898444

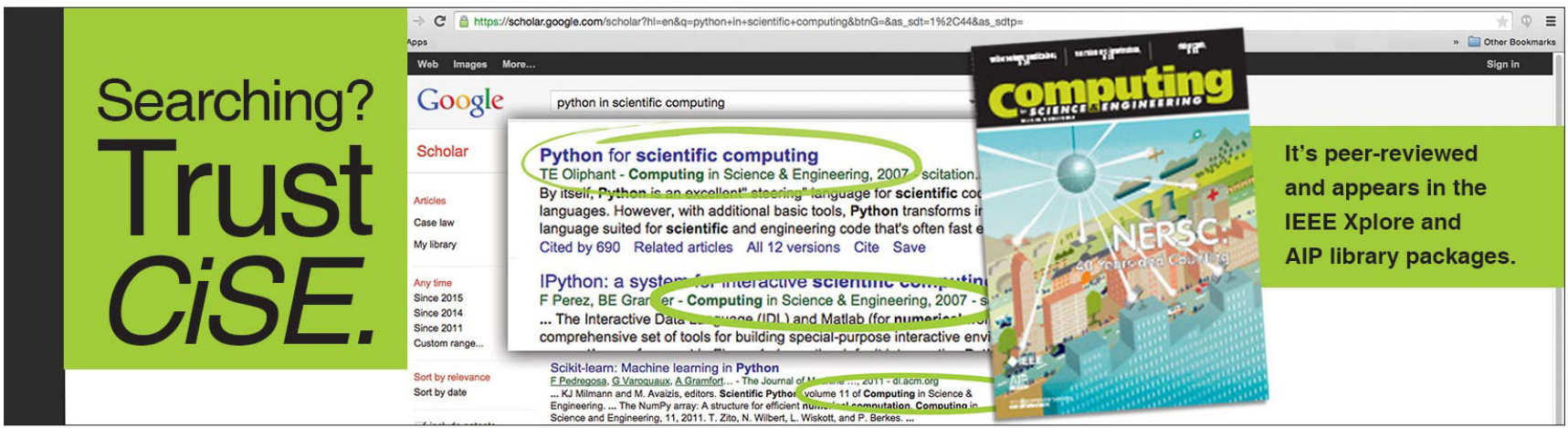




\title{
Laser material processing with tightly focused cylindrical vector beams
}

\author{
Rokas Drevinskas, ${ }^{1, a)}$ Jingyu Zhang, ${ }^{1}$ Martynas Beresna, ${ }^{1}$ Mindaugas Gecevičius, ${ }^{1}$ \\ Andrey G. Kazanskii, ${ }^{2}$ Yuri P. Svirko, ${ }^{2,3}$ and Peter G. Kazansky ${ }^{1}$ \\ ${ }_{1}^{1}$ Optoelectronics Research Centre, University of Southampton, Southampton SO17 1BJ, United Kingdom \\ ${ }^{2}$ Physics Department, M.V. Lomonosov Moscow State University, Moscow 119991, Russia \\ ${ }^{3}$ Institute of Photonics, University of Eastern Finland, P.O.BOX 111, FI-80101 Joensuu, Finland
}

(Received 27 April 2016; accepted 21 May 2016; published online 3 June 2016)

\begin{abstract}
We demonstrate a comprehensive modification study of silica glass, crystalline silicon, and amorphous silicon film, irradiated by tightly focused cylindrical vector beams with azimuthal and radial polarizations. The evidence of the longitudinal field associated with radial polarization is revealed by second harmonic generation in $z$-cut lithium niobate crystal. Despite the lower threshold of ring-shaped modification of silicon materials, the modification in the center of single pulse radially polarized beam is not observed. The phenomenon is interpreted in terms of the enhanced reflection of longitudinal component at the interface with high-index contrast, demonstrating that the longitudinal component is inefficient for the flat surface modification. Enhanced interaction of the longitudinal light field with silicon nanopillar structures produced by the first pulse of double-pulse irradiation is also demonstrated. Published by AIP Publishing. [http://dx.doi.org/10.1063/1.4953455]
\end{abstract}

The radially polarized beams focused with high numerical aperture optics lead to strong longitudinal electric fields, which are attractive for many applications, including particle acceleration, ${ }^{1}$ high capacity optical recording, ${ }^{2}$ near-field microscopy, ${ }^{3}$ optical trapping of metallic particles, ${ }^{4}$ and second-harmonic (SH) generation. ${ }^{5-7}$ However, refractive index mismatch at the interface can strongly affect the light field that oscillates perpendicular to the surface. ${ }^{8,9}$ Specifically, the field is suppressed when the beam propagates from a low refractive index into a high refractive index medium. This implies that it may be difficult to employ the field component perpendicular to the surface in the laser processing of materials with high refractive indices.

Successful ablation experiments of borosilicate glass by the single or multi-pulse irradiation ${ }^{10}$ and modification of silicon with relatively high-index by the multi-pulse irradiation with the pronounced longitudinal field were reported. ${ }^{11}$ Furthermore, enhanced modification by an electric field component perpendicular to the surface via a resonant absorption mechanism ${ }^{12}$ was suggested to explain the results of the laser-induced nickel ablation. ${ }^{13}$ Nevertheless, the direct experimental evidence confirming that the normal field component could produce stronger surface ablation was not demonstrated.

The vectorial distribution of the electric field could be visualized via self-assembled nanogratings, which are always aligned perpendicular to the electric field of the incident beam. ${ }^{10,14-16}$ Tailored optical vector beams including radially and azimuthally polarized could be also exploited for complex surface structuring with ultrashort light pulses. ${ }^{17}$

\footnotetext{
${ }^{\text {a) }}$ Author to whom correspondence should be addressed. Electronic mail: rd1c12@soton.ac.uk
}

In this letter, using amorphous silicon (a-Si:H), we demonstrate that the longitudinal component of the single pulse tightly focused radially polarized beam is inefficient for modification of flat surface. On the other hand, double pulse experiments indicate that the same vector beams exhibit an improved micromachining performance when employed for processing of pillar-like nanostructures on a crystalline silicon surface. The interaction of tightly focused radially and azimuthally polarized beams at the interfaces with different index contrast is experimentally analyzed for both bulk and thin film materials including silica glass, crystalline silicon and amorphous silicon. The experimental results agree well with the analytical and numerical simulations. In addition, to verify the presence of a strong longitudinal field, the total field of tightly focused radially and azimuthally polarized beams is indirectly visualized by second harmonic patterns generated in a z-cut lithium niobate $\left(\mathrm{LiNbO}_{3}\right)$ crystal. Moreover, the longitudinal electric field of the focused radially polarized light beam is revealed by the characterization of the self assembled nanogratings inside silica glass.

The experiments were performed with a femtosecond laser system (Pharos, Light Conversion, Ltd.) operating at a wavelength of $1030 \mathrm{~nm}$ and delivering pulses of $270 \mathrm{fs}$ at repetition rates of $20 \mathrm{kHz}$ (for silicon materials) and $200 \mathrm{kHz}$ (for silica glass and lithium niobate crystal). The lower repetition rate was used to eliminate any possible heat accumulation in silicon between the first and second pulses. The longitudinal component was generated by tightly focusing radially polarized laser beam, using 0.65 NA dry objectives and 1.2 NA water immersion objectives (Fig. 1).

The Fourier computer generated hologram $(\mathrm{CGH})$ that converts the initial Gaussian beam into the target annular beam was generated using the adapted weighted Gerchberg-Saxton (GSW) algorithm. ${ }^{18}$ The beam with 


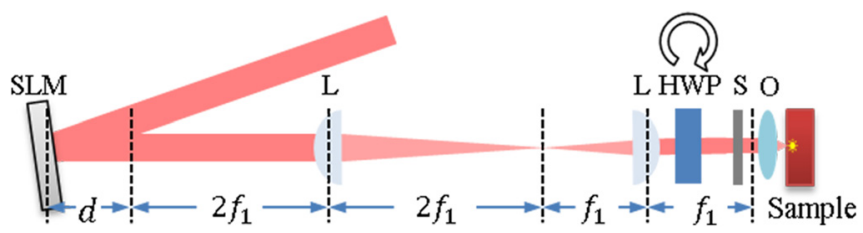

FIG. 1. Setup for femtosecond laser direct writing. Spatial light modulator (SLM), lenses (L), half-wave plate (HWP), S-waveplate (S), objective lens $(\mathrm{O}) ; d$ is the distance necessary for the beam to propagate after the reflection from SLM, and $2 f_{1}, f_{1}$ correspond to the focal lengths of lenses with $f_{1}=300 \mathrm{~mm}$.

annular intensity distribution shaped with an electrically addressed spatial light modulator (SLM) (Hamamatsu, LCOS-SLM) was used to enhance the longitudinal component. ${ }^{2,19,20}$ The Keplerian beam expander was used to adjust the beam size to match the pupil size of the objective lenses. The cylindrical distribution of the electric field polarization was controlled using a spatially variant polarization converter ${ }^{21}$ referred to as $\mathrm{S}$-waveplate. The radially or azimuthally polarized laser beams are generated by rotating the orientation of the incident linear polarization via a half-wave plate placed before the S-waveplate.

The electric field distribution of the focused annular cylindrical vector beams at the focal region is defined as follows: ${ }^{22}$

$$
\begin{aligned}
& \text { Radial : } E_{\text {transverse }}(r, z) \\
& =A \int_{\beta}^{\alpha} \sqrt{\cos (\theta)} \sin (2 \theta) l(\theta) J_{1}(k r \sin (\theta)) e^{i k z \cos (\theta)} d \theta,
\end{aligned}
$$

$$
E_{z}(r, z)=2 i A \int_{\beta}^{\alpha} \sqrt{\cos (\theta)} \sin ^{2}(\theta) l(\theta) J_{0}(k r \sin (\theta)) e^{i k z \cos (\theta)} d \theta,
$$

Azimuthal: $E_{\text {transverse }}(r, z)$

$$
=2 A \int_{\beta}^{\alpha} \sqrt{\cos (\theta)} \sin (\theta) l(\theta) J_{1}(k r \sin (\theta)) e^{i k z \cos (\theta)} d \theta,
$$

where $r$ and $z$ are cylindrical coordinates, $\alpha$ and $\beta$ are the focusing angles determined by the outer and inner radius of the annular shaped beam, and $l(\theta)$ is the relative amplitude of the field. If the non-annular shapes are used, the $\beta$ value becomes zero. In the case of $\beta / \alpha \approx 0.5$ and uniform relative amplitude, the ratio of the generated longitudinal and transverse light intensities in immersion medium is around 0.58 for $0.65 \mathrm{NA}$ dry objective and 2.5 for 1.2 NA water immersion objective. Such focusing conditions were used in all following experiments and simulations.

Longitudinal field does not propagate and is confined to the focal region making its observation very difficult. However, longitudinal component can be visualized via a second harmonic generation. The second harmonic (SH) patterns generated by radially and azimuthally polarized beams can be obtained using the following second-order polarization $P(2 \omega)$ generated in the $z$-cut $\mathrm{LiNbO}_{3}$ crystal $^{23,24}$

$$
\left(\begin{array}{c}
P_{x}(2 \omega) \\
P_{y}(2 \omega) \\
P_{z}(2 \omega)
\end{array}\right)=\left(\begin{array}{cccccc}
0 & 0 & 0 & 0 & d_{31} & -d_{22} \\
-d_{22} & d_{22} & 0 & d_{31} & 0 & 0 \\
d_{31} & d_{31} & d_{33} & 0 & 0 & 0
\end{array}\right)\left(\begin{array}{c}
E_{x}^{2} \\
E_{y}^{2} \\
E_{z}^{2} \\
2 E_{y} E_{z} \\
2 E_{x} E_{z} \\
2 E_{x} E_{y}
\end{array}\right),
$$

for azimuthal :

$$
\begin{aligned}
& P_{x}(2 \omega)=-2 d_{22} E_{x} E_{y}, \\
& P_{y}(2 \omega)=-d_{22} E_{x}^{2}+d_{22} E_{y}^{2},
\end{aligned}
$$

and for radial :

$$
\begin{aligned}
& P_{x}(2 \omega)=-2 d_{22} E_{x} E_{y}+2 d_{31} E_{x} E_{z}, \\
& P_{y}(2 \omega)=-d_{22} E_{x}^{2}+d_{22} E_{y}^{2}+2 d_{31} E_{y} E_{z},
\end{aligned}
$$

where $E_{x, y, z}$ are Cartesian components of the electric field of the fundamental beam introduced in Eqs. (1)-(3), and $d_{22}=2.1 \mathrm{pm} / \mathrm{V}, d_{31}=4.3 \mathrm{pm} / \mathrm{V}$, and $d_{33}=27 \mathrm{pm} / \mathrm{V}$ are the absolute values of components of nonlinear susceptibility tensor. $^{25}$

In order to verify the existence of the longitudinal field, the cylindrical vector beams were focused inside a $z$-cut lithium niobate crystal via a 0.65 NA microscope objective $100 \mu \mathrm{m}$ beneath the surface. The generated SH was collected with a 1.2 NA water immersion objective after the sample, imaging the focal plane and far-field intensity distributions of the focusing lens. The SH patterns were observed for both cylindrical radially and azimuthally polarized beams, while the pronounced longitudinal component in the radially polarized beam results in $\sim 70 \%$ higher $\mathrm{SH}$ power (at $1.5 \mathrm{TW} / \mathrm{cm}^{2}$ pump intensities) in comparison with that produced by the azimuthally polarized beam. The $\mathrm{SH}$ power increase is caused by the $2 d_{31} E_{x} E_{z}$ and $2 d_{31} E_{y} E_{z}$ components of the second-order polarization produced by the non-zero longitudinal $\left(E_{z}\right)$ component of the field at the fundamental frequency (Eq. (4)). When the longitudinal field is enhanced, e.g., the ratio with transverse field is 0.58 , the contribution of these terms in $\mathrm{SH}$ generation increases.

The focal plane SH pattern has the shape of an annular ring for both radial and azimuthal polarizations (see Figs. 2(b-iii) and 2(d-iii)), while in the far-field $\mathrm{SH}$ beam profile becomes polarization dependent. Specifically, if the fundamental beam is azimuthally polarized, the far-field $\mathrm{SH}$ beam remains ring-shaped (Fig. 2(b-vi)), while the radially polarized fundamental beam in the far-field evolves into a threelobed pattern (Fig. 2(d-vi)), which is determined by the orientation of the $X$ and $Y$ axes in the $z$-cut lithium niobate crystal. These experimental observations agree very well with the results of numerical simulation shown in Figs. 2(a) and 2(c), where the polarization component $P_{z}(2 \omega)=d_{31}$ $\left(E_{x}{ }^{2}+E_{y}{ }^{2}\right)+d_{33} E_{z}{ }^{2}$ is not considered because it does not contribute to the transversal SH field measured in our experiments. One can conclude that the difference in the transverse $\mathrm{SH}$ patterns generated by the focused radially and azimuthally polarized beams originate from the longitudinal component of the fundamental light field. It should be noted that since the Figure 2 shows only transversal component of the SHG wave, the observable quantitative difference in the 
Focal plane

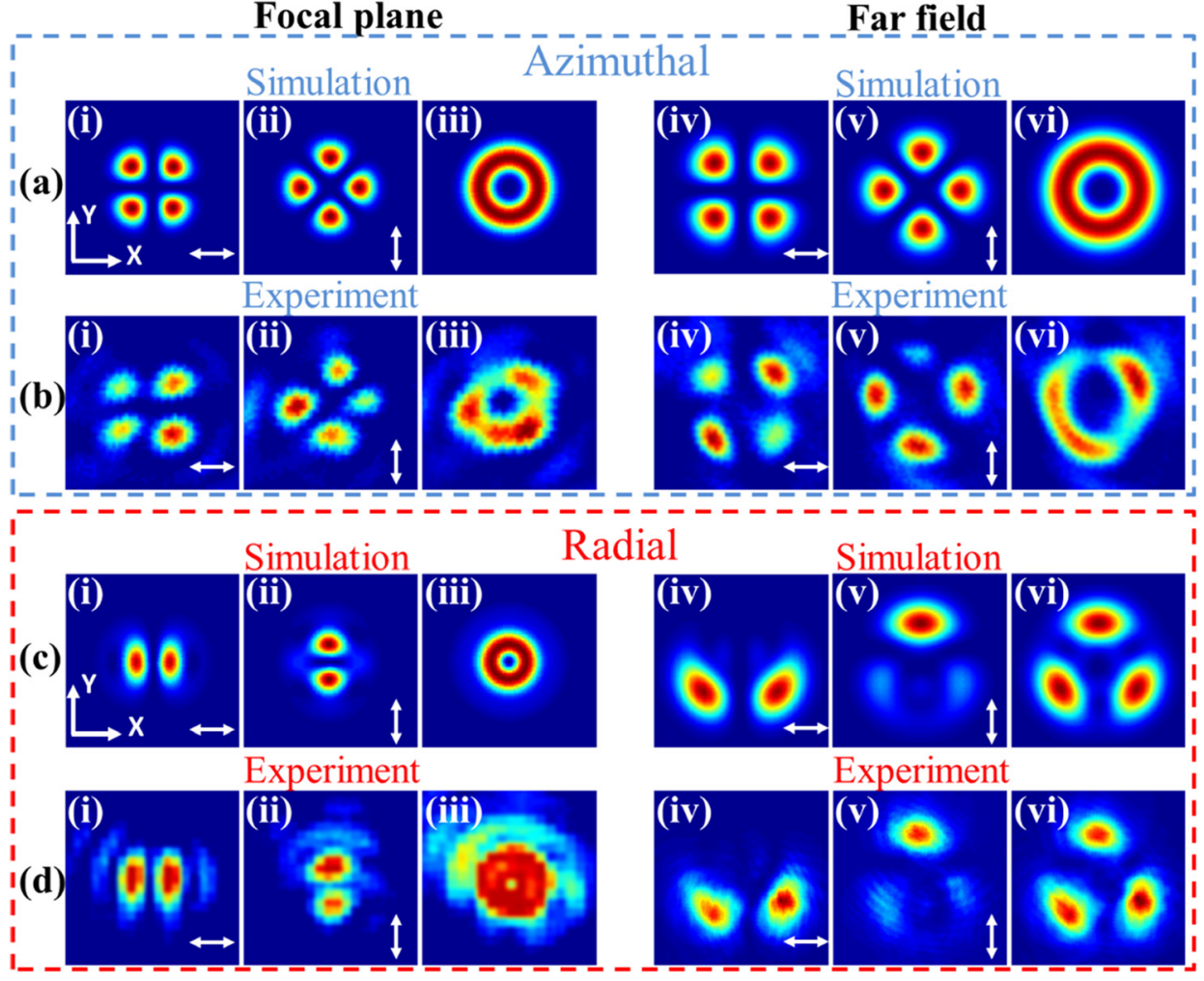

FIG. 2. Second-harmonic patterns generated by focusing ((a) and (b)) azimuthally and ((c) and (d)) radially polarized laser beams, (i, ii, iii) imaged focal plane and (iv, v, vi) the far-field of the focusing lens. In simulations, only the transverse component is shown. White arrows indicate the polarization state of light after the linear analyzer. The $x$ and $y$ axes show the crystal orientation. All intensities are normalized.
SHG intensity calculated (iii-c) and measured (iii-d) originates from the nonzero longitudinal component, whichaccording to Eq. (4) - is nonzero in the focal plane. As well, the imaging performed with the high numerical aperture optics makes the measurements very sensitive to any minor deviations in the system that can affect the signal.

In further experiments, single dots were imprinted inside silica glass to visualize the vectorial distribution of electric field in the focus. A train of 2000 pulses with $250 \mathrm{~nJ}$ pulse energy was focused via a 0.65 NA objective, $120 \mu \mathrm{m}$ beneath the substrate surface. Under these conditions, the selfassembled nanogratings are induced. The nanostructures with the sub-wavelength periodicity behave as a uniaxial birefringent material where the slow axis is oriented perpendicular to the beam polarization. The slow axis orientation with corresponding vectorial distribution of electric field was mapped using quantitative birefringence measurement system (CRi Abrio; Olympus BX51) (Fig. 3).

The birefringence cannot be observed if the optical axis is oriented perpendicular to the image plane (Fig. 3(a-iii)). Thus, the slow axis induced by the longitudinal field can be visualized only from the side of the structure. One can observe from Fig. 3(b-iii) that the center of the light-induced modification is occupied by an anisotropic structure (light beam) having a slow axis (polarization) oriented perpendicular (parallel) to the beam propagation direction. However, on the sides of the modified region, the nanogratings with orthogonal orientation are induced, which reveals the transverse component of the light polarization (Fig. 3(b-iv)). In contrast, after irradiation with the azimuthally polarized light, the anisotropy associated with longitudinal component is not observed (Fig. 3(b-i)).

Further, to directly imprint the intensity distribution of the tightly focused annular cylindrically polarized beams in high-index material, we carried out the experiments on irradiation of $300 \mathrm{~nm}$ thick a-Si:H film. The water immersion microscope objective of 1.2 NA was used for focusing of the beam. The irradiation with pulse energies below $150 \mathrm{~nJ}$ were ensured to induce the nanocrystalline type of modification without ablation in amorphous silicon. ${ }^{26,27}$ In total, the 21 sets of dots were imprinted by a single pulse irradiation of radially or azimuthally polarized beams at different focusing depths ranging from $-10 \mu \mathrm{m}$ to $10 \mu \mathrm{m}(0.5 \mu \mathrm{m}$ step $)$, relative to the substrate surface (Fig. 4). By choosing the strongest modification, we ensure that the modification is performed in the focus (Fig. 4(i), (ii)).

Weak dependence of the modification on the focus depth was observed indicating a Bessel-like long Rayleigh length, typical for cylindrically polarized beams (Fig. 4). The threshold energy of modification was more than $40 \%$ lower for

(a)
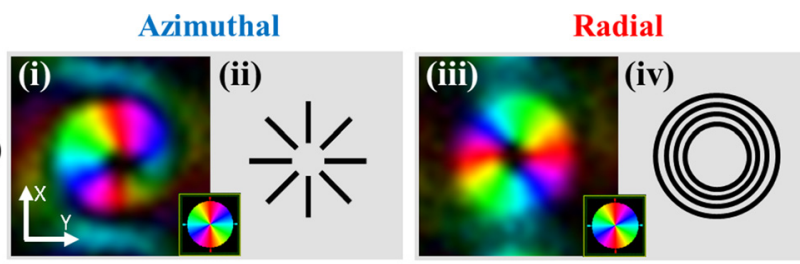

(b)

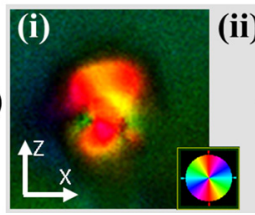

(ii)

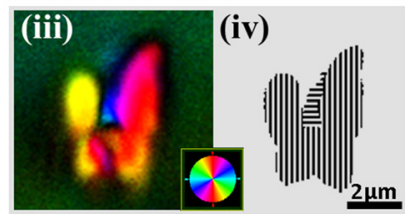

FIG. 3. Slow axis characterization of the birefringent structures inside silica glass induced by azimuthally (left) and radially (right) polarized laser beams. (a) (i, iii) Top view and (b) (i, iii) side view of the slow axis orientation, with (a) (ii, iv), (b) (ii, iv) their corresponding schematics of the possible nanogratings distribution. Pseudo color indicates the direction of slow axis (insets). 


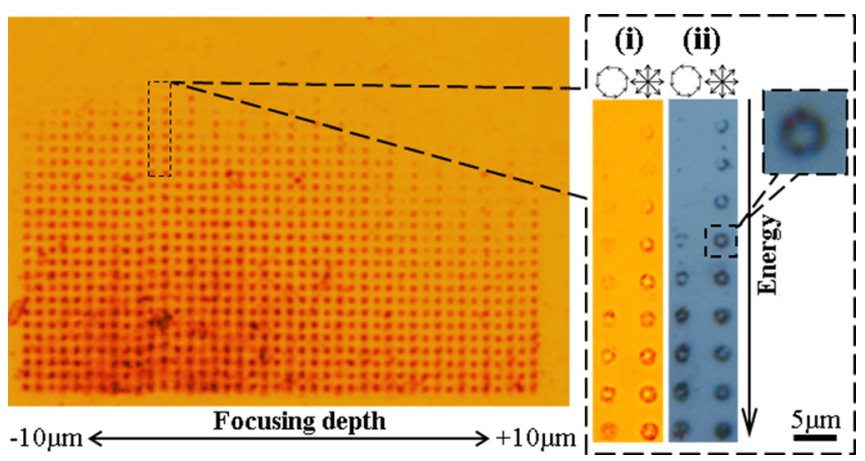

FIG. 4. Optical transmission images of structures imprinted by annularshape of radially and azimuthally polarized beams in amorphous silicon film at different focus planes, where in the inset the same structures are compared in transmission (i) and reflection (ii) modes. The pulse energy in the inset (right images) varies from $30 \mathrm{~nJ}$ to $70 \mathrm{~nJ}$.

radially polarized than for azimuthally polarized beams, $30 \mathrm{~nJ}$ versus $50 \mathrm{~nJ}$, respectively. This phenomenon could be explained by the lower Fresnel reflection and different material transformation dynamics for $p$-polarization compared to $s$-polarizations at the interface of modified region. ${ }^{28}$ However, in both cases, only the ring-shaped patterns were produced. The modification in the center of annulus, where the strong longitudinal field is present, was not observed. The surface of the film remains flat in the conditions of irradiation below the ablation threshold. Consequently, if the second pulse is fired, modification of the film qualitatively will be the same as by the first pulse and will not reveal the presence of longitudinal component.

Finally, we performed experiments on ablation of $p$-type crystalline silicon (111). The radially and azimuthally polarized beams with pulse energy of $150 \mathrm{~nJ}$ were focused on the surface via a $1.2 \mathrm{NA}$ water immersion objective. The irradiation by the single pulse radially polarized beam resulted in a ring-shaped modification with nanopillar structure in the center (Fig. 5 (top-left)). Specifically, the molten silicon is pushed away radially from the region with maximum deposited energy leading to the ring-shaped ablation pattern. The excess material pushed to the center of the ring solidifies forming the nanopillar structure. In the case of azimuthal polarization, the nanopillar structure is not formed due to the lack of deposited energy and possibly the different material dynamics after irradiation. ${ }^{28}$ The deposited energy is suppressed by the higher Fresnel reflection for $s$-polarization (azimuthal case) compared to $p$-polarization (radial case) at the interface.

The laser-induced nanopillar, which was emerged along the beam propagation direction, was subsequently ablated during the second pulse irradiation due to the enhanced transmission of the longitudinal field along the distorted interface (Fig. 5 (top-right)). However, the irradiation by double pulse azimuthally polarized beam kept the center untouched partially covering it with the ablated material (Fig. 5 (bottom)). This observation indicates that the needle shaped surface structures aligned along the beam propagation direction are necessary for interaction with the longitudinal field.

Numerical simulations of focused vector beams were performed $^{29}$ to understand why the strong effect of
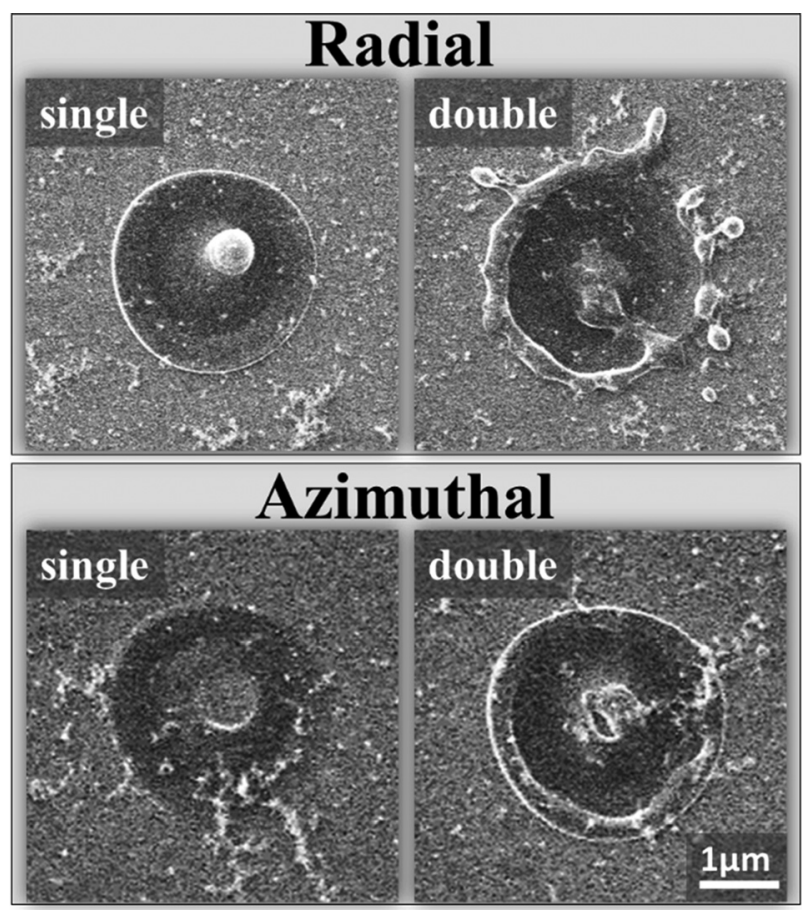

FIG. 5. SEM images of the laser processed crystalline silicon surface. Each spot was irradiated with single (left) and double (right) pulse radially (top) and azimuthally (bottom) polarized beams with energies of $150 \mathrm{~nJ}$.

longitudinal field did not produce modification in amorphous and crystalline silicon. The simulations revealed that the transverse electric field propagates without significant amplitude changes, while the longitudinal electric field is discontinuous through the interface when the incident beam is focused at the water/silicon interface (Fig. 6 (top)). The magnitude of longitudinal component decreases by a factor of $\varepsilon_{2} / \varepsilon_{1}(\sim 7.4)$, where $\varepsilon_{1}$ and $\varepsilon_{2}$ are the dielectric constants of the media. Moreover, the total intensity distribution of radially and azimuthally polarized beams after the transmission through the water/silicon interface (Fig. 6 (top)) match well with the structures imprinted in the amorphous silicon film (Fig. 4).

On the other hand, inside the silica glass, the magnitude of longitudinal and transverse components are of the same order, since the refractive index contrast at the air/silica interface is relatively low (Fig. 6 (bottom)). Experimentally, it was confirmed by the nanogratings formation both parallel and perpendicular to the beam propagation direction (Fig. 3).

In conclusion, we have demonstrated the evidence of longitudinal component of tightly focused radially polarized beam by the observation of enhanced second harmonic generation in the $z$-cut lithium niobate crystal and by the laserinduced anisotropy oriented perpendicular to the longitudinal field in silica glass. Despite the lower threshold of ring-shaped modification in amorphous silicon film and the nanopillar formation in crystalline silicon, the longitudinal electric field generated by the single pulse radially polarized beam produces a negligible modification, which can be explained by the discontinuity of longitudinal field at the high-index-contrast interfaces. Thus, the longitudinal electric field is not effective for modifying flat surfaces of materials with high refractive index when irradiating from a low index 


\section{Focused Radially Polarized Beam}
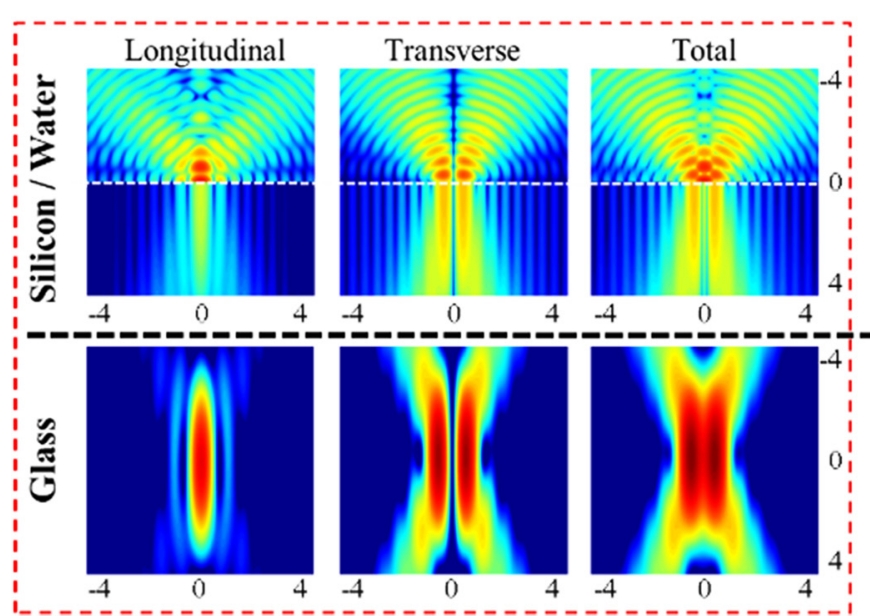

I. - - - - -

mediums. On the other hand, the effect of the high-indexcontrast interface can be eliminated by using high numerical aperture immersion objectives, e.g., solid immersion lenses. Alternatively, the sub-wavelength structures oriented along the beam propagation direction such as nanopillars or enhanced surface roughness could be beneficial for material processing by pronounced longitudinal field.

The study has been supported by EPSRC (Grant No. EP/ M029042/1). P.G.K. thanks Ministry of Education and Science of the Russian Federation (Grant No. 14.Z50.31.0009). Y.P.S. thanks Russian Foundation for Basic Research (Grant No. 16-02-00684). The data for this work are accessible through the University of Southampton Institutional Research Repository (http://dx.doi.org/10.5258/SOTON/384580).

${ }^{1}$ Y. Liu, D. Cline, and P. He, Nucl. Instrum. Methods Phys. Res. A 424, 296 (1999).

${ }^{2}$ X. Li, Y. Cao, and M. Gu, Opt. Lett. 36, 2510 (2011).

${ }^{3}$ M. R. Beversluis, L. Novotny, and S. J. Stranick, Opt. Express 14, 2650 (2006).

${ }^{4}$ Q. Zhan, Opt. Express 12, 3377 (2004).

${ }^{5}$ A. Ohtsu, Y. Kozawa, and S. Sato, Appl. Phys. B Lasers Opt. 98, 851 (2010).

${ }^{6}$ D. P. Biss and T. G. Brown, Opt. Lett. 28, 923 (2003).

${ }^{7}$ G. Bautista, M. J. Huttunen, J. Mäkitalo, J. M. Kontio, J. Simonen, and M. Kauranen, Nano Lett. 12, 3207 (2012).

${ }^{8}$ G. M. Lerman and U. Levy, Opt. Express 16, 4567 (2008).

${ }^{9}$ W.-C. Kim, Y.-J. Yoon, H. Choi, N.-C. Park, and Y.-P. Park, Opt. Express 16, 13933 (2008)

${ }^{10}$ C. Hnatovsky, V. G. Shvedov, W. Krolikowski, and A. Rode, Phys. Rev. Lett. 106, 123901 (2011).
Focused Azimuthally

Polarized Beam

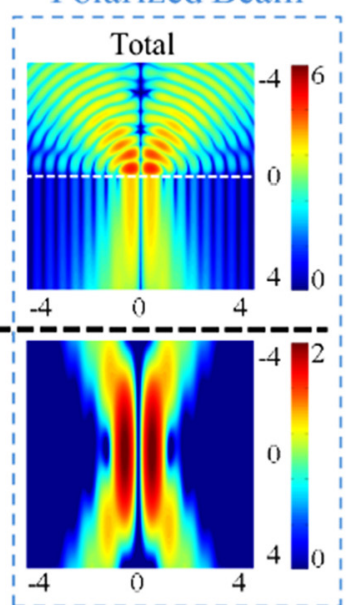

FIG. 6. Simulation of cylindrical vector beams focused on silicon surface ( $n=3.613$ ) (top) and inside silica glass $(n=1.458)$ (bottom) via a $1.2 \mathrm{NA}$ water-immersion $(n=1.3265)$ microscope objective (top) and via a 0.65 NA dry microscope objective (bottom). Color scales are logarithmic in arbitrary units; axes are in the units of wavelength.
${ }^{11}$ C. Hnatovsky, V. G. Shvedov, N. Shostka, A. V Rode, and W. Krolikowski, Opt. Lett. 37, 226 (2012).

${ }^{12}$ F. Brunel, Phys. Rev. Lett. 59, 52-55 (1987).

${ }^{13}$ H. Iwase, S. Kokubo, S. Juodkazis, and H. Misawa, Opt. Express 17, 4388-4396 (2009).

${ }^{14}$ P. G. Kazansky, H. Inouye, T. Mitsuyu, K. Miura, J. Qiu, K. Hirao, and F. Starrost, Phys. Rev. Lett. 82, 2199 (1999).

${ }^{15}$ Y. Shimotsuma, P. G. Kazansky, J. Qiu, and K. Hirao, Phys. Rev. Lett. 91, 247405 (2003).

${ }^{16}$ B. Poumellec, M. Lancry, A. Chahid-Erraji, and P. G. Kazansky, Opt. Mater. Express 1, 766-782 (2011).

${ }^{17}$ J. Ouyang, W. Perrie, O. J. Allegre, T. Heil, Y. Jin, E. Fearon, D. Eckford, S. P. Edwardson, and G. Dearden, Opt. Express 23, 12562-12572 (2015).

${ }^{18}$ R. Di Leonardo, F. Ianni, and G. Ruocco, Opt. Express 15, 1913-1922 (2007).

${ }^{19}$ L. Yang, X. Xie, S. Wang, and J. Zhou, Opt. Lett. 38, 1331 (2013).

${ }^{20}$ H. Wang, L. Shi, B. Lukyanchuk, C. Sheppard, and C. T. Chong, Nat. Photonics 2, 501 (2008).

${ }^{21}$ M. Beresna, M. Gecevičius, P. G. Kazansky, and T. Gertus, Appl. Phys. Lett. 98, 201101 (2011).

${ }^{22}$ K. Youngworth and T. Brown, Opt. Express 7, 77 (2000).

${ }^{23}$ G. D. Boyd, R. C. Miller, K. Nassau, W. L. Bond, and A. Savage, Appl. Phys. Lett. 5, 234 (1964).

${ }^{24}$ P. A. Franken, A. E. Hill, C. W. Peters, and G. Weinreich, Phys. Rev. Lett. 7, 118 (1961).

${ }^{25}$ D. A. Roberts, IEEE J. Quantum Electron. 28, 2057 (1992).

${ }^{26}$ A. V. Emelyanov, A. G. Kazanskii, M. V. Khenkin, P. A. Forsh, P. K. Kashkarov, M. Gecevicius, M. Beresna, and P. G. Kazansky, Appl. Phys. Lett. 101, 081902 (2012).

${ }^{27}$ R. Drevinskas, M. Beresna, M. Gecevičius, M. Khenkin, A. G. Kazanskii, I. Matulaitienè, G. Niaura, O. I. Konkov, E. I. Terukov, Yu. P. Svirko, and P. G. Kazansky, Appl. Phys. Lett. 106, 171106 (2015).

${ }^{28}$ Q. Zhang, Adv. Opt. Photonics 1, 1-57 (2009).

${ }^{29}$ D. Biss and T. Brown, Opt. Express 9, 490 (2001). 